\title{
INVESTIGACIONES
}

\section{La cátedra de la paz como eje de desarrollo social de cara al posconflicto}

\author{
The peace chair as an axis of social development \\ in the post-conflict era
}

\author{
Kodalys Toro Osorio ${ }^{a}$,Tulio Amaya De Armas $^{b}$, Carmen Romero Zúñiga \\ ${ }^{a}$ Institución Educativa San Rafael Ovejas, Colombia. \\ kodatoro@hotmail.com \\ ${ }^{b}$ Facultad de Educación de la Universidad Católica de la Santísima Concepción, Chile. \\ tuama1@hotmail.com \\ ${ }^{c}$ Universidad de La Guajira, Colombia. \\ carmenmilagro@uniguajira.edu.co
}

\section{RESUMEN}

Se reporta un trabajo realizado con 176 estudiantes, 30 profesores y 16 padres de familia de tres instituciones educativas oficiales de una población colombiana que fue sede de los diálogos de paz que pusieron fin al conflicto con un grupo guerrillero. Se estudiaron tópicos relacionados con la implementación de la cátedra de la paz y se analizó el grado de acuerdo entre ellos respecto a estos tópicos. Para el procesamiento de la información se hizo un análisis de correspondencias múltiples con el Chi cuadrado de Pearson como coeficiente de prueba, y además, se hicieron grupos focales con miembros de cada estamento. Los resultados muestran que el proceso de implementación de la cátedra de la paz en las instituciones educativas, ha sido lento, con algunas dificultades, con retraso en su implementación de más de dos años, y falta de capacitación y acompañamiento, por parte de las entidades territoriales, a las comunidades de las instituciones educativas. Pero a pesar de las dificultades dentro del contexto escolar se fomenta la cultura del diálogo para resolver los conflictos que se presentan, y con los profesores como mediadores en el proceso. La actividad escolar se da en un clima de respeto y apoyo mutuo entre directivos, docentes, estudiantes y padres de familia.

Palabras claves: cultura de paz, post-conflicto, cátedra de la paz, desarrollo social, cultura del diálogo.

\begin{abstract}
A work with 176 students, 30 teachers and 16 parents from three official educational institutions of a Colombian population that hosted the peace dialogues that ended the conflict with a guerrilla group is reported. For the information processing, a multiple correspondence analysis was made with Pearson's Chi square as a test coefficient, and focus groups were also made with members of each estate. The results show that the process of implementation of the chair of peace in educational institutions has been slow, with some difficulties, delayed in its implementation of more than two years, and lack of training and accompaniment, by the entities territorial, to the communities of educational institutions. But despite the difficulties within the school context, the culture of dialogue is encouraged to resolve conflicts that arise, and with teachers as mediators in the process. The school activity takes place in a climate of respect and mutual support between managers, teachers, students and parents.

Key words: culture of peace, post-conflict, chair of peace, social development, culture of dialogue.
\end{abstract}




\section{INTRODUCCIÓN}

La sociedad colombiana ha sido víctima de la violencia, producto del conflicto armado que ha azotado a este territorio por más de cinco décadas. Flagelos como el secuestro, el desplazamiento forzado, las masacres y toda clase de violación a los derechos humanos, han dejado una huella en la historia del país, así como en la memoria de los habitantes, que han sobrevivido en medio de tantas situaciones adversas. Hoy, cuando nos encontramos frente a una salida negociada al conflicto armado, con los grupos -FARC y ELN- se han planteado diversas estrategias, encaminadas a favorecer los procesos de reconciliación y resocialización, necesarias para avanzar hacia el posconflicto: entre ellas la educación.

La educación se convierte en un escenario propicio para fomentar la cultura de la paz a través de la formación de los niños, niñas y adolescentes, que serán los futuros ciudadanos y líderes del país. Este proceso supone, un cambio de actitud para solucionar los conflictos de manera pacífica y el fortalecimiento de los valores humanos, entre ellos la justicia, la tolerancia, el respeto a la diferencia, la solidaridad y la responsabilidad social, elementos necesarios para asegurar una convivencia armónica. Uno de los objetivos de la Educación es la Paz, es el desarrollo de las personas y el mejoramiento de su calidad de vida, por lo que los establecimientos educativos deben contribuir a generar procesos sociales basados en la confianza, la solidaridad y el respeto mutuo, que ayuden a pensar de una forma nueva las relaciones humanas (Organización de las Naciones Unidas para la Educación, la Ciencia y la Cultura [UNESCO], 2005). En la escuela, los niños y adolescentes tienen una posibilidad única de desarrollar las competencias necesarias para integrarse y contribuir a consolidar una sociedad democrática, justa y solidaria (Fondo de las Naciones Unidas para la Infancia [UNICEF], 2002), donde puedan crecer y desarrollar al máximo sus potencialidades sociales, que los habiliten para adaptarse a un mundo cada vez más globalizado.

Partiendo de esta realidad, en Colombia durante los últimos años se ha generado una serie de iniciativas estatales encaminadas a favorecer la cultura de la paz desde el ámbito educativo, convirtiendo a la educación en el eje articulador para el desarrollo social y económico del país. La Cátedra de la Paz, es una de las políticas implementadas para generar ambientes pacíficos desde las aulas. La implementación de ésta se ha planteado como una asignatura de obligatorio cumplimiento en las instituciones educativas colombianas. Según el Ministerio de Educación Nacional,

La cátedra deberá fomentar el proceso de apropiación de conocimientos y competencias relacionadas con el territorio, la cultura, el contexto económico y social y la memoria histórica; con el propósito de reconstruir el tejido social, promover la prosperidad general y garantizar la efectividad, los principios, derechos y deberes consagrados en la Constitución $(2015$, p. 3).

La Cátedra de la Paz se concibe entonces, como una estrategia para abrir espacios de diálogo al interior de los establecimientos educativos que permitan crear una cultura de paz y disminuir de este modo los niveles de intolerancia, de violencia y discriminación. Esta cátedra se convierte en un instrumento para mitigar o disminuir los índices de violencia y preparar a los jóvenes y a la sociedad en general para una cultura de paz, y de este modo, enfrentar el postconflicto (Ospina, 2010; González, 2019). 
La paz vista desde el conflicto armado de un país es un tema sensible y la implementación de políticas encaminadas hacia su construcción, conlleva grandes dificultades, razón por la cual se hace necesario identificar la manera como se aborda la articulación de estas políticas en los escenarios reales. Hace más de dos décadas, al respecto Galtung (1996) hacía una afirmación que hoy en día sigue vigente:

el tratamiento del conflicto por medios no violentos y creativos es crucial para lograr la paz y eso requiere profundizar en la cultura y estructura social, donde se origina el conflicto, como mejor forma de prevenir y, en su caso, resolver los brotes de violencia (p. 2).

Así las cosas, el presente trabajo investigativo permitió analizar en contexto, la manera cómo la cátedra de la paz ha generado transformaciones en la praxis social e individual, manifestada en la vivencia de los valores y el respeto de los derechos humanos al interior de las comunidades educativas. Se indagó si la realidad social, está acorde con los valores para la construcción de una cultura de paz y de respeto. A partir de este análisis se pudo generar un proceso de reflexión en torno a las estrategias implementadas para consolidar una paz estable y duradera que permita superar las huellas de la violencia desde los escenarios educativos.

\section{APROXIMACIÓN TEÓRICA}

El conflicto es considerado como el núcleo principal de la educación para la paz, y no puede entenderse como una batalla a ganar o perder, sino como una problemática a ser resuelta con apertura y equidad a los intereses de todas las partes (Fisas, 2006). La educación para la paz surge para dar una respuesta a la crisis del mundo, en la cual el ser humano se agrede a sí mismo, agrede a los demás y a la naturaleza (Cerdas, 2013). Es una propuesta ético-política integral, que promueve la autonomía y la emancipación de personas y colectivos, dotándolas de competencias para participar crítica y democráticamente en los diversos procesos de transformación social, orientados a la construcción de sociedades más justas e igualitarias (Ospina, 2010). Por ende, es una educación que no se enfoca sólo en contenidos, sino que posibilita la acción y el desarrollo del pensamiento crítico, dotando a sus participantes de habilidades para la transformación de los conflictos por medios pacíficos (Cabezudo, 2012).

De lo anterior se puede inferir que la educación para la paz no intenta evitar los conflictos, sino más bien formar individuos capaces de resolverlos por medios no violentos (Ospina, 2010). Dentro de este escenario, los conflictos se convierten en un reto, una oportunidad de crecimiento, de desarrollo personal, de cambio y transformación, en la excusa perfecta para poner en evidencia las competencias que permitan su tratamiento a través del diálogo y la negociación; y su solución, en un proceso de aceptación, tanto del otro y sus diferencias, como de sí mismo, que permita una sana convivencia socialmente compartida entre pares.

De acuerdo con Pérez-Sauceda (2015), en el marco de la educación para la paz, la resolución pacífica de conflictos se reafirma como la mejor estrategia, y la escuela como el mejor escenario de formación para la paz. De ahí que el educador como el orientador 
del proceso educativo por naturaleza, debe convertirse en un enlace entre las necesidades de sus estudiantes y los recursos existentes en su entorno, ofreciendo a sus alumnos la ayuda necesaria para que éstos comprendan, organicen, amplíen y desarrollen habilidades cognitivas y emocionales que les permitan abordar y afrontar situaciones y conflictos cotidianos (Parra, 2014).

La educación para la paz debe estar integrada en el currículum como uno de los ejes vertebrales de una práctica educativa (Ospina, 2010). Pues es en los escenarios educativos, donde se desarrollan los procesos de formación y se potencia la construcción de sociedades democráticas. No obstante, de acuerdo con los planteamientos de Cerdas (2013), la estructura del sistema educativo se convierte en el mayor obstáculo con el que se enfrenta la educación para la paz, al ser una estructura violenta en sí misma. Según esta autora, la función social del sistema educativo actual es contraria a los valores que persigue la educación para la paz, en cuanto que fomenta la competitividad, el individualismo, la xenofobia, el conformismo y la pasividad.

Hoy día la educación es la principal reproductora de violencia y discriminación, por lo que es necesaria una transformación de las estructuras escolares y de los procesos de construcción democrática al interior de los establecimientos educativos (Ospina, 2010). Según Parra (2014), la escuela debe ser inclusiva y participativa tanto en su modelo pedagógico como de gestión. Debe generar un clima de seguridad, de confianza, de apoyo mutuo, de esta manera los conflictos que se presenten tendrán más posibilidades de ser resuelto de forma participativa en este contexto de democracia y colaboración comunitaria.

La educación para la paz es un derecho y una necesidad dentro del proceso educativo y debería comenzar construyendo relaciones de paz entre todos los miembros de las comunidades (Jarés, 1999; Ospina, 2010), como una necesidad de la vida misma. Así, la familia y la sociedad deberían ser los principales responsables de la iniciación de este proceso (Ospina, 2010; González, 2019), para posteriormente, continuarlo en las aulas de clase a partir de la creación de comunidades de comunicación, en la que todos los seres humanos son igualmente reconocidos como interlocutores, válidos capaces de moderar sus comportamientos (Martínez, 2001). Comportamientos que deben ajustándose a los requerimientos sociales, que les faciliten transformar los conflictos en acciones socialmente aceptadas por los miembros de la comunidad donde habitan.

El hecho de aceptarse y aceptar al otro le permite al individuo, el desarrollo de competencias ciudadanas (Sandoval, 2014), y tales competencias, en el marco de la formación para una ciudadanía responsable, se abren campo como un elemento trasversal dentro del currículo educativo, el cual debe permear, todas las esferas de actuación de la escuela. Hoy en día la formación ciudadana es un tema de importancia, relevante dentro de la sociedad y de modo particular en las instituciones educativas, puesto que las personas deben aprender a comportarse de acuerdo a unas normas sociales que se comparten al vivir en comunidad (Redon, 2010).

Teniendo en cuenta lo anterior, y que vivimos en un mundo cada vez más globalizado, mediado por el uso de las tecnologías de la información y la comunicación, centrado en la competitividad, con dinámicas sociales complejas, se hace necesaria la formación de un ciudadano competente que pueda desenvolverse eficazmente en su contexto con responsabilidad social (Ruiz, Jaraba y Romero, 2005). Es decir, se requieren ciudadanos cumplidores de sus deberes, de la ley y el orden, buscadores y propiciadores de la paz, participativos, críticos, defensores de los derechos propios y respetuosos de los de los demás. 
Las propuestas de formación ciudadana al interior de las instituciones educativas deben ser coherentes con estas demandas sociales, generando espacios reales de interacción y participación, donde el estudiante pueda aprender a convivir con otros, aceptando la diferencia y respetando la diversidad (Cerdas, 2013; Cabezudo, 2012; Parra, 2014), pero teniendo en cuenta la familia y los demás miembros de la comunidad, que faciliten una gran armonía social.

La Cátedra de la Paz, es una apuesta del Estado colombiano para favorecer la construcción de una cultura de paz, a través de la apropiación de conocimientos y competencias que permitan la reconstrucción del tejido social, el ejercicio de una ciudadanía responsable y el mejoramiento de la calidad de vida de la población (Ministerio de Educación Nacional, 2014). Abordar los antecedentes de la Cátedra de la Paz, obliga a remitirse a un universo conceptual más amplio del cual surge este término, como proyecto pedagógico: haciendo referencia a la educación para la paz y la cultura de paz.

La escuela y el colegio, concebidos como lo público, se constituyen en escenarios para llevar a cabo procesos dinámicos hacia el logro de intereses comunes y de convivencia basada en la comunicación efectiva como escenario de encuentro, en detrimento de la coacción y de la violencia. Estas instituciones le dan a la sociedad la oportunidad de convertir los derechos de los otros en los propios deberes y viceversa porque "lo público se encuentra en el rostro del otro" (Hoyos, 2008).

Para Delle Donne (2018), el concepto central de la formación en ciudadanía es el de la responsabilidad, no sólo el de hacerse responsable de sus propias decisiones sino aprender a crear condiciones para que todos puedan verse protegidos en sus derechos, aunque no estén en situación de hacerlo por sí mismos. La formación ciudadana tiene como misión entregar herramientas capacidades, normas y valores para que los alumnos una vez adultos, se hagan parte activa en la sociedad donde viven aprendiendo a interactuar en una sociedad cambiante (Sacristán, 2001).

De acuerdo con Macip (2014), la formación ciudadana promueve el desarrollo de las siguientes competencias,

- Sentido de justicia y legalidad, fundamentado en la práctica del valor de la igualdad y la dignidad humana, reconociendo el valor de la norma y su importancia dentro de la convivencia armónica.

- Valoración de la democracia como forma de vida y de gobierno, entendiendo las relaciones existentes entre la democracia, las leyes, las instituciones y la participación ciudadana.

Respeto a la diferencia y rechazo a la discriminación, entendida como el irrespeto o desaprecio a la diversidad, en lo propuesto por Macip no queda explicito el respeto y la auto aceptación de cada uno, como principio fundamental para respetar, aceptar y comprender al otro, y así llegar a procesos convencionales que faciliten la convivencia social. 


\section{METODOLOGIA}

En esta investigación se siguió un enfoque mixto (Johnson y Onwuegbuzie, 2004; Creswell, 2009) combinando técnicas y métodos de investigación cuantitativos y cualitativos. Atendiendo el enfoque cuantitativo, se cuantificaron para su estudio, las opiniones de estudiantes, padres de familia y docentes en relación a los diferentes tópicos relacionados con la implementación de la cátedra de la paz en tres instituciones educativas oficiales colombianas, y se analizó el grado de acuerdo entre ellos respecto a estos tópicos. Para cuantificarlas se crearon cinco categorías, donde cada número expresa el grado de acuerdo del participante con la implementación del constructo por el que se indaga: 1 indica que está totalmente en desacuerdo, 2 que está en desacuerdo y 3 imparcialidad (ni de acuerdo, ni en desacuerdo), 4 que está de acuerdo y 5 indica que está totalmente de acuerdo, al responder la cuestión por la que se le indagó. A partir de esta convención, el tratamiento que, en adelante, se hizo de estas variables es meramente cuantitativo.

Desde el enfoque cualitativo, se indaga por las complejas relaciones que encierra el contexto escolar, en cuanto al desarrollo de las competencias ciudadanas, generadas por la implementación de la cátedra de la paz, las cuales se hicieron evidentes en la convivencia escolar, a partir de la interacción con las personas implicadas en el proceso.

Se hizo un estudio descriptivo de casos (Servan y Serván, 2010), el cual permitió describir el proceso de implementación de la cátedra de la paz en contextos particulares y su impacto al interior de las comunidades educativas. Se enfatizó en las observaciones basadas más en informes descriptivos que en categorías pre-establecidas, interesados por describir la conducta observada, dentro del marco de los hechos circundantes, y la perspectiva de los participantes acerca de los hechos, es decir, cómo construyen su realidad social (Cebreiro y Fernández, 2004), y cómo se ven en el marco del postconflicto.

La población objeto de este estudio la constituyeron docentes, estudiantes de educación media y padres de familia de tres instituciones educativas colombianas, de una comunidad que fue escenario el proceso de paz con un grupo armado. Para la muestra se seleccionaron 176 estudiantes $\left(\mathrm{I}_{1}: 72, \mathrm{I}_{2}: 53\right.$ y $\mathrm{I}_{3}: 50$, es decir, un grado completo del nivel de enseñanza media de cada institución educativa); 30 docentes $\left(\mathrm{D}_{1}: 10, \mathrm{D}_{2}: 10\right.$ y $\left.\mathrm{D}_{3}: 10\right)$ y 16 padres de familia $\left(\mathrm{P}_{1}: 4, \mathrm{P}_{2}: 4\right.$ y $\left.\mathrm{P}_{3}: 4\right)$, donde $\mathrm{I}_{\mathrm{i}}, \mathrm{D}_{\mathrm{i}}$ y $\mathrm{P}_{\mathrm{i}}$ representa la institución i-ésima, el número de docentes y de padres de familia de dicha institución. Tanto las instituciones, como los grupos y los individuos, fueron seleccionados a través de un muestreo aleatorio simple, utilizando el método coordinado negativo como mecanismo de solución. Se aplicó un cuestionario escala tipo Likert y se hicieron grupos focales, para los que se seleccionó a 10 estudiantes, 10 docentes y 10 padres de familia para integrarlos.

Para el diseño de los cuestionarios, se hizo necesario operacionalizar las categorías de análisis, estableciendo definiciones conceptuales observables dentro de cada variable, mediante la definición de indicadores que permitieran bajar su nivel de abstracción e hicieran posible observarla en la realidad. Los indicadores que se midieron a través de esta técnica fueron: importancia de la cátedra de la paz, estrategias de solución de conflictos, promoción y defensa de los derechos humanos, inclusión, promoción de la sana convivencia, participación democrática y pluralidad.

El cuestionario está compuesto por 50 ítems: 5 ítems para medir el grado de conceptualización logrado por los participantes sobre educación para la paz, 14 para medir el grado de implementación de la cátedra de la paz, 17 para medir la armonía en la 
convivencia y en la solución de conflictos en la comunidad y 14 para medir los niveles de apropiación en lo relacionado con competencias ciudadanas y derechos humanos por parte de los participantes.

Para el procesamiento de la información cuantitativa se hizo: (1) un análisis de correspondencia múltiples, con tablas de contingencia y el coeficiente Chi cuadrado de Pearson $\left(c^{2}\right)$ como prueba para validar las asociación entre el grado de conceptualización que han logrado los participantes sobre educación para la paz, las competencias ciudadanas alcanzadas luego de la implementación de la Cátedra de la Paz, la armonía en la convivencia y en la solución de conflictos en la comunidad y los niveles de apropiación en lo relacionado con competencias ciudadanas y derechos humanos, con el estamento institucional al que pertenecieran los grupos. (2) Se calculó el coeficiente Alfa de Cronbach con el que se analizó la fiabilidad del instrumento. En la tabla 1 se muestra un resumen de tal proceso, donde se indica que dicho instrumento tiene una buena consistencia interna (García, González y Ballesteros, 2001) y, 3) se hizo un análisis factorial para analizar la validez de constructo y el comportamiento psicométrico de las características de las variables, con lo que se determinó que éstas en su conjunto representan adecuadamente el concepto estudiado $(\mathrm{KMO}=0.81, \mathrm{P}<0.05)$.

Tabla 1. Resumen del análisis de fiabilidad del cuestionario

\begin{tabular}{|l|c|c|}
\hline Constructo & Alfa de Cronbach & N de elementos \\
\hline Grado de conceptualización & 0.825 & 5 \\
\hline $\begin{array}{l}\text { Competencias ciudadanas luego de la } \\
\text { implementación de la catedra de la paz }\end{array}$ & 0.846 & 14 \\
\hline Convivencia armónica y solución de conflictos & 0,781 & 17 \\
\hline $\begin{array}{l}\text { Niveles de apropiación sobre competencias } \\
\text { ciudadanas y derechos humanos }\end{array}$ & 0.813 & 14 \\
\hline Total & 0.876 & 50 \\
\hline
\end{tabular}

Para el procesamiento de la información de los grupos focales se utilizó la técnica análisis de contenido (Bernárdez, 1995), tanto para las categorías previas como para las categorías inductivas.

\section{RESULTADOS Y ANÁLISIS}

\subsection{CONCEPCIONES SOBRE EDUCACION PARA LA PAZ}

Al indagar con los diferentes estamentos sobre el concepto de educación para la paz, se encontró alto grado de acuerdo intragrupos $\left(\mathrm{c}^{2}=16,643, \mathrm{P}<0,05\right)$ sobre la claridad conceptual de este término. Las opiniones de docentes y padres de familia, asocian este concepto a la práctica de valores, al cumplimiento de normas de convivencia, a la solución 
pacífica de conflictos y la formación ciudadana, orientada fundamentalmente a favorecer la convivencia armónica. Por otro lado, los estudiantes la asocian directamente con el conflicto armado, asumiéndola como un proceso de reconciliación, en el cual las víctimas de la violencia perdonan a sus victimarios y estos s su vez se comprometen a la no repetición del conflicto. De igual forma coinciden al afirmar, que la educación para la paz es la principal garantía de no repetición del conflicto, en la medida en que debe favorecer la comprensión de las causas que dieron origen a más de medio siglo de violencia generalizada. En este sentido, coincidimos con Fierros (2013) en que, a través de la comprensión de la realidad política y social del país, de discusiones en torno a los acuerdos de paz, el rescate de valores y el establecimiento de una convivencia armónica que ponga fin a tantos odios y resentimientos dejados por el conflicto armado, tanto victimas como victimarios aprenderán a utilizar las palabras en vez de las armas de fuego. En la tabla 2 se muestra un resumen de los resultados por estamentos del grado de conceptualización logrado sobre cátedra de la paz, y también el correspondiente chi cuadrado de Pearson.

Tabla 2. Resumen del grado de conceptualización logrado sobre catedra de la paz

\begin{tabular}{|c|c|c|c|c|c|}
\hline & \multicolumn{3}{|c|}{ Grado de conceptualización } & \multirow[b]{2}{*}{ Total } \\
\hline & & $\begin{array}{c}\text { Me es } \\
\text { indiferente }\end{array}$ & $\begin{array}{c}\text { De } \\
\text { acuerdo }\end{array}$ & $\begin{array}{l}\text { Totalmente } \\
\text { de acuerdo }\end{array}$ & \\
\hline \multirow{3}{*}{$\begin{array}{l}\text { Rol dentro de la } \\
\text { Comunidad Educativa }\end{array}$} & Estudiante & 28 & 123 & 25 & 176 \\
\hline & Padre de familia & 4 & 6 & 6 & 16 \\
\hline & Docente & 6 & 13 & 11 & 30 \\
\hline \multicolumn{2}{|l|}{ Total } & 38 & 142 & 42 & 222 \\
\hline \multirow{2}{*}{\multicolumn{2}{|c|}{ Chi Cuadrado de Pearson }} & $\mathrm{gl}$ & Sig. & \multicolumn{2}{|c|}{ Valor } \\
\hline & & 4 & 0,004 & \multicolumn{2}{|c|}{$c^{2}=15,572$} \\
\hline
\end{tabular}

Si bien es cierto que la educación para la paz surge como una propuesta a la negación del uso de la violencia, para solucionar los conflictos, ésta trasciende del concepto de no violencia, hacia el establecimiento de una cultura de paz, entendida como aquella que pone fin a la injusticia, la inequidad, la discriminación y la exclusión (Bueno-Cipagauta, 2017; Jojoa, 2016), por lo que entenderla podría ayudar en la consolidación de una cultura de paz en estas comunidades tan golpeadas por el conflicto armado.

En relación a los temas que debería abordar la educación para la paz, los miembros de las comunidades educativas consideran relevante el tema de los valores, pero trascendiendo de la conceptualización, a la práctica de éstos dentro de escenarios reales. Al respecto, coincidieron con Padilla (2016), en que hay que enfatizar en el establecimiento de normas de comportamientos que orienten la sana convivencia y el buen trato, se debe trabajar también el diálogo como estrategia de solución pacifica de conflicto, así como los derechos humanos y el respeto a la diferencia. 
Estas apreciaciones guardan mucha coherencia con el planteamiento de Cabezudo (2012) quien afirma que la educación para la paz, no se enfoca sólo en contenidos, sino que posibilita la acción y el desarrollo del pensamiento crítico, dotando a sus participantes de habilidades para la transformación de los conflictos por medios pacíficos. Todo esto es de suma importancia en el desarrollo del proceso, sin embargo, los participantes en sus respuestas excluyen la participación democrática, que según Castillo (2003), es un elemento fundamental en la educación para la paz. Es decir, no parecen visionarse como protagonistas en sus propios procesos de crecimiento endógeno.

Todos los estamentos consideraron pertinente la implementación de la asignatura Cátedra de Paz, porque ayuda a la reconstrucción de la memoria histórica, se trabajan los valores que conllevan a una convivencia pacífica y se favorece el reconocimiento de los derechos humanos. No obstante, la mayoría de ellos considera que si bien la asignatura afianza y potencia conocimientos asociados a la paz es insuficiente para generar una cultura de paz por si sola. Ya que dentro de este proceso, inciden factores como el entorno familiar y el contexto social donde se desenvuelven los sujetos. En palabras de Jojoa (2016) la construcción de una cultura de paz es un proceso que exige compromisos a muy largo plazo, de todos los actores y en todos los niveles de nuestra sociedad. Por lo que no es responsabilidad exclusiva de los profesores que orientan la asignatura, sino de todos los miembros de la comunidad.

De acuerdo con Bueno-Cipagauta (2017), el establecimiento de una cultura de paz se logra repensando y rediseñando no sólo el sistema educativo, para crear comunidades de aprendizaje orientadas por la búsqueda de la verdad, el respeto mutuo, la participación democrática, el respeto a la diferencia y el aprendizaje de mecanismos de solución pacífica de conflictos. Según esta autora, además de lo anterior, se logra, rediseñando el estado y las instituciones públicas para que encarnen enfoques diferenciales e inclusivos, la descentralización del poder y la superación de los regionalismos. Pero también, minimizando la desigualdad social, el analfabetismo, la pobreza extrema y la corrupción en los órganos del Estado.

\subsection{DESARROLLO DE COMPETENCIAS CIUDADANAS A PARTIR DE LA IMPLEMENTACIÓN DE LA CÁTEDRA DE PAZ}

Para identificar las competencias ciudadanas generadas en los estudiantes a partir de la implementación de la Cátedra de la Paz. Los diferentes estamentos coinciden en afirmar $\left(c^{2}=12,200, \mathrm{P}<0,05\right)$ que, luego de la implementación de la Cátedra de la Paz, se hacen evidentes cambios de comportamiento positivos, en el trato respetuoso a los demás, el uso del diálogo para solucionar los conflictos, el control de las emociones y el comportamiento dentro de las instituciones, aunque consideran que es prematuro valorar transformaciones a mayor escala por el desarrollo incipiente de la asignatura. Si bien la Cátedra de la Paz ha hecho énfasis en el uso de estrategias de solución pacífica de conflictos, la presencia de agresiones dentro los contextos educativos eran escasas. Situación que obedecen a diferentes estrategias pedagógicas que se habían estado desarrollando en las instituciones antes de su implementación. En la tabla 3 se muestra un resumen de los resultados por estamentos, sobre el grado de competencia ciudadana alcanzado a partir de la implementación de la Cátedra de la Paz, y además, el correspondiente Chi cuadrado de Pearson. 
Tabla 3. Resumen de los resultados sobre grado de competencias ciudadana adquiridas en el desarrollo de la Cátedra de la Paz

\begin{tabular}{|c|c|c|c|c|c|}
\hline & \multicolumn{3}{|c|}{$\begin{array}{l}\text { Competencias ciudadanas a partir de la } \\
\text { cátedra de la paz }\end{array}$} & \multirow{2}{*}{ Total } \\
\hline & & $\begin{array}{c}\text { Me es } \\
\text { indiferente }\end{array}$ & $\begin{array}{c}\text { De } \\
\text { acuerdo }\end{array}$ & $\begin{array}{l}\text { Totalmente } \\
\text { de acuerdo }\end{array}$ & \\
\hline \multirow{3}{*}{$\begin{array}{l}\text { Rol dentro de la } \\
\text { Comunidad Educativa }\end{array}$} & Estudiante & 39 & 115 & 22 & 176 \\
\hline & Padre de familia & 6 & 5 & 5 & 16 \\
\hline & Docente & 9 & 13 & 8 & 30 \\
\hline \multicolumn{2}{|l|}{ Total } & 54 & 133 & 35 & 222 \\
\hline \multirow{2}{*}{\multicolumn{2}{|c|}{ Chi Cuadrado de Pearson }} & gl & Sig. & \multicolumn{2}{|c|}{ Valor } \\
\hline & & 4 & 0,016 & \multicolumn{2}{|c|}{$c^{2}=12,200$} \\
\hline
\end{tabular}

Sin embargo, hubo bastante heterogeneidad en las respuestas de los participantes en relación al fomento de la pluralidad y respeto a la diferencia. Algunos manifiestan que en su institución se evidencian formas de discriminación y exclusión, y el trato que reciben los miembros de las comunidades educativas no es igualitario, pero que desde la cátedra de la paz se favorece el reconocimiento y respeto de los derechos humanos.

En relación con la presencia de formas de discriminación y exclusión en el contexto educativo, docentes y padres de familia coinciden en afirmar que estas son nulas, puesto que en las instituciones se promueve el respeto a las diferencias individuales sea de carácter cultural, religioso o étnico. No obstante, los estudiantes manifiestan que entre algunos de ellos es frecuentes la discriminación al otro por su aspecto físico o rendimiento académico. Esto se evidencia en burlas, la exclusión a la hora de formar grupos de trabajo y objeciones al expresar sus opiniones. Así mismo, expresan que son evidentes preferencias por parte de algunos educadores, hacia los estudiantes que presentan mejor rendimiento académico y buen comportamiento. Estos comportamientos muestran que aún no se consolida una cultura de paz en estas comunidades, pues, no han logrado trascender el concepto de no violencia, que los lleve a respetarse y a aceptarse como son, para vivir armónicamente en comunidad (Bueno-Cipagauta, 2017; Jojoa, 2016).

Al indagar por el reconocimiento de los derechos humanos por parte de la comunidad educativa, los estamentos coinciden en afirmar que, aunque la cátedra ha profundizado el estudio de los derechos humanos, esta temática se venía desarrollando a través de otras asignaturas como lo son Ciencias sociales y ética. En cuanto al conocimiento previo sobre participación democrática, los miembros de la comunidad educativa asocian este concepto con la libre expresión de opiniones y la participación en la toma de decisiones haciendo, uso de diferentes mecanismos y estrategias, entre ellas el voto popular, respetando los diferentes puntos de vista. Estas apreciaciones coinciden con el planteamiento de Rodríguez y Arango (2017) quien considera la participación democrática como la libertad y/o autonomía que tiene cada ciudadano para decidir y tomar partido frente a las decisiones que les afecten, pensando en el bienestar colectivo, llegando a consensos u acuerdos que promuevan el 
diálogo de intereses. Los acuerdos entre los diferentes estamentos, evidencian claridad conceptual en estos tópicos, y manifiestan que las instituciones propician instancias que los favorecen. No obstante, consideran que, aunque las instituciones ofrecen a los diferentes estamentos, espacios de participación a través de los diferentes órganos del gobierno escolar y los comités de las áreas de gestión, la incidencia de éstos en la toma de decisiones es mínima, lo que según Delle Donne (2018) desfavorece las actitudes hacia la responsabilidad, con sus propias decisiones, el establecimiento y reclamo de sus derechos, que los vaya poniendo en condición de valerse por sí mismos.

Sin embargo, vale la pena resaltar que las instituciones educativas lograron solo, hasta el año 2017, la articulación de la cátedra de la paz como asignatura dentro del plan de estudios, cuando el plazo establecido por el Estado, para tal fin, de acuerdo con lo previsto en el decreto 1038 de 2015 era el 31 de diciembre de 2015. Los docentes manifiestan falta de conocimiento de la ley 1732 de 2014, (que crea la Cátedra de la Paz) y su decreto reglamentario 1038 de 2015. A pesar de que los educadores tenían conocimiento de la existencia de la cátedra de la paz, a nivel institucional y municipal no se dieron espacios para su discusión y apropiación por parte del colectivo docente de las instituciones del sector mayormente afectado por el conflicto, por ser zona de asentamiento de los diálogos de paz. En este sentido, se hizo una única jornada de capacitación con asistencia solo de profesores de ciencias sociales, como si fuera responsabilidad de éstos, y no de todos, materializar esta iniciativa dentro del currículo institucional. Los profesores de ciencias sociales dicen que no fue una capacitación, fue un encuentro donde se les entregó unas cartillas orientadoras en las que debían apoyarse para la implementación de la asignatura. Comentan, que esta acción resultó insuficiente para suplir sus vacíos conceptuales en materia de derechos humanos, cultura de paz, competencias ciudadanas, participación democrática, diversidad y pluralidad, lo que ha generado que muchas de las temáticas y objetivos consagrados en el decreto reglamentario no hayan sido abordados desde la asignatura.

Lo anterior deja ver que es una experiencia muy joven aún, que podría considerarse en proceso experimental, que su implementación ha sido bastante lenta, con muchas dificultades, pero lo más importante es que ya está en funcionamiento, y que ha tenido buena acogida por parte de las comunidades educativas afectadas por el conflicto.

\subsection{CONVIVENCIA ARMÓNICA Y SOLUCION DE CONFLICTOS}

Los diferentes estamentos muestran acuerdos $\left(\mathrm{c}^{2}=10,006, \mathrm{P}<0,05\right)$ en la forma como se resuelven los conflictos en las instituciones educativas, éstos coinciden en afirmar que se, abordan pacíficamente a través del diálogo y la mediación, y como dice Parra (2014) en la mayoría de los casos son los docentes los que actúan como mediadores entre los estudiantes. Éstos, a través de un dialogo dirigido, escuchan las partes implicadas y les ayudan a encontrar una solución concertada, aunque en ocasiones la solución es planteada por el docente dejando inconforme a alguna de las partes. Cuando no es posible una solución a través de la mediación del docente y dependiendo la gravedad del conflicto, éste puede ser remitido al comité de convivencia escolar, quien evalúa los hechos y toma una decisión al respecto, imponiendo sanciones de ser necesarias. No obstante, cuando se llega a estas instancias algunos de los encuestados expresan inconformidad con las decisiones del comité, al no considerarlas justas y equitativas. En la tabla 4 se muestra un resumen de los resultados por estamentos sobre la forma de resolver los conflictos, y además, el correspondiente Chi cuadrado de Pearson. 
Tabla 4. Resumen de la forma de resolver los conflictos, según la opinión de los diferentes estamentos

\begin{tabular}{|l|l|c|c|c|c|}
\hline \multicolumn{2}{|c|}{} & \multicolumn{3}{|c|}{$\begin{array}{c}\text { Convivencia armónica y solución de } \\
\text { conflictos }\end{array}$} & \multirow{2}{*}{ Total } \\
\cline { 3 - 6 } & $\begin{array}{c}\text { Me es } \\
\text { indiferente }\end{array}$ & $\begin{array}{c}\text { De } \\
\text { acuerdo }\end{array}$ & $\begin{array}{c}\text { Totalmente } \\
\text { de acuerdo }\end{array}$ & \\
\hline \multirow{3}{*}{$\begin{array}{l}\text { Rol dentro de la } \\
\text { Comunidad Educativa }\end{array}$} & Estudiante & 46 & 112 & 18 & 176 \\
\cline { 2 - 6 } & Padre de familia & 5 & 11 & 0 & 16 \\
\cline { 2 - 6 } & Docente & 1 & 24 & 5 & 30 \\
\hline \multirow{2}{*}{\begin{tabular}{l} 
Total \\
\multirow{2}{*}{ Chi Cuadrado de Pearson }
\end{tabular}} & 52 & 147 & 23 & 222 \\
\cline { 3 - 6 } & & 4 & gl & Sig. & \multicolumn{2}{|c|}{ Valor } \\
\hline
\end{tabular}

A pesar de los avances logrados por la comunidad de la mano de las instituciones, parecen no tener claro que resolver un conflicto de manera pacífica supone superar la visión de ganar a toda costa, por encima del otro, estar abierto al diálogo, al acuerdo y la escucha activa, tener la capacidad para reconocer las razones y sentimientos del otro, considerando nuevas posibilidades en las que todas las partes resulten ganadoras en el proceso de resolución (Parra, 2016).

\subsection{APROPIACIÓN DE COMPETENCIAS CIUDADANAS Y DERECHOS HUMANOS}

Los resultados muestran altos niveles de acuerdo $\left(\mathrm{c}^{2}=15,560, \mathrm{P}<0,05\right)$ en cuanto al grado de apropiación de competencias ciudadanas y derechos humanos entre los participantes. La caracterización de la convivencia escolar a partir de la implementación de la cátedra de la paz, la determinan indicadores como la promoción de la sana convivencia, la inclusión, la prevención y mitigación de la violencia. Para lograrlo según Padilla (2016), es necesario enfatizar en el diálogo, el respeto a la diferencia y a los derechos del otro, como estrategia de solución pacifica de conflictos, que faciliten el establecimiento de normas de comportamientos que orienten la sana convivencia y el buen trato. En la tabla 5 se muestra un resumen de los resultados por estamentos del nivel de apropiación logrado sobre competencias ciudadanas y derechos humanos, y también del correspondiente chi cuadrado de Pearson. 
Tabla 5. Resumen del nivel de apropiación manifiesto sobre competencias ciudadanas y derechos humanos de los participantes

\begin{tabular}{|c|c|c|c|c|c|c|}
\hline & & \multicolumn{4}{|c|}{ Competencias ciudadanas y derechos humanos } & \multirow[b]{2}{*}{ Total } \\
\hline & & $\begin{array}{c}\text { En } \\
\text { desacuerdo }\end{array}$ & $\begin{array}{l}\text { Me es } \\
\text { indiferente }\end{array}$ & $\begin{array}{c}\text { De } \\
\text { acuerdo }\end{array}$ & $\begin{array}{l}\text { Totalmente } \\
\text { de acuerdo }\end{array}$ & \\
\hline \multirow{3}{*}{$\begin{array}{l}\text { Rol dentro de } \\
\text { la Comunidad } \\
\text { Educativa }\end{array}$} & Estudiante & 3 & 39 & 110 & 24 & 176 \\
\hline & Padre de familia & 0 & 1 & 15 & 0 & 16 \\
\hline & Docente & 1 & 0 & 22 & 7 & 30 \\
\hline \multicolumn{2}{|l|}{ Total } & 4 & 40 & 147 & 31 & 222 \\
\hline \multirow{2}{*}{\multicolumn{2}{|c|}{ Chi Cuadrado de Pearson }} & $\mathrm{gl}$ & Sig. & \multicolumn{3}{|c|}{ Valor } \\
\hline & & 6 & 0,016 & \multicolumn{3}{|c|}{$c^{2}=15,560$} \\
\hline
\end{tabular}

Si bien, en todas las instituciones educativas se encuentra conformado un comité de convivencia escolar, este se dedica principalmente a la atención de conflictos entre los diferentes miembros de la comunidad educativa, dejando de lado el componente de prevención de la violencia y promoción de la sana convivencia definido en la ruta de atención integral a la convivencia escolar. De acuerdo con Hernández (2016), la escuela debe fomentar en niños y jóvenes el desarrollo de valores, actitudes, comportamientos y habilidades que promuevan la no violencia, en el marco del respeto y cumplimiento de los derechos humanos. Y esto es posible a través de la implementación de estrategias orientadas hacia el cambio de actitudes violentas por relaciones pacíficas que posibiliten la construcción de una cultura de paz, en otras palabras, la promoción de la sana convivencia.

En lo referente al tema de la inclusión, se puede afirmar que, aunque las instituciones educativas no cuenten con una estrategia clara de inclusión, definida dentro de su proyecto educativo institucional, tratan de brindar un trato equitativo e igualitario a los diferentes miembros de la comunidad educativa. De acuerdo con la UNESCO (2005), la inclusión está asociada al reconocimiento de las habilidades y potencialidades propias de cada persona, es un proceso que atiende y responde a la diversidad, a las diferencias individuales, e involucra la participación reciproca de todos los miembros de una comunidad.

Al indagar si dentro de la convivencia escolar se evidencian acciones excluyentes, algunos estudiantes consideran que aún son visibles formas de discriminación y exclusión por parte de un número reducido de alumnos que aíslan a sus compañeros por sus rasgos físicos o desempeños académicos. Aunque cada vez son menos, en la medida en que estas prácticas son desaprobadas por el colectivo docente y estudiantil.

Con la implementación de la cátedra, se ha generado un espacio importante para abordar con mayor libertad y profundidad las temáticas asociadas a la educación para la paz y para crear ambientes de aprendizaje propicios para el desarrollo de competencias ciudadanas. Ahora bien, es necesario aprovecharlo al máximo y no limitarla al manejo de contenidos que, si bien son importantes, no son lo fundamental. En el tránsito hacia una cultura de paz, es prioritario hacer énfasis en el desarrollo de habilidades comunicativas, emocionales, cognitivas e integradoras que posibiliten una convivencia armónica, se 
requieren cambios estructurales que potencien el conocimiento de la memoria historia y cultural y la participación ciudadana como estrategia fundamental para la construcción de una sociedad en paz.

Aunque la implementación de la cátedra de la paz ha contribuido a abordar el estudio de temáticas asociadas a la cultura de paz, no ha generado una transformación de fondo en los contextos escolares. Su puesta en escena no ha trascendido de ser una asignatura más del currículo. Es urgente proponer su trabajo interdisciplinar, garantizar la formación docente sobre el tema, y el diseño de una estrategia pedagógica que permita articular acciones para dinamizar su trabajo y generar un mayor impacto, considerando la importancia que tienen esta asignatura para avanzar hacia el establecimiento de una cultura de paz.

\section{CONCLUSIONES}

De los resultados obtenidos en el presente estudio se puede concluir que el proceso de implementación de la cátedra de la paz en las instituciones educativas ha sido lento, con algunas dificultades, con retraso en su implementación de más de dos años, y falta de capacitación y acompañamiento, por parte de las entidades territoriales, a las comunidades instituciones educativas. Estas dificultades podrían estar afectando el tratamiento de elementos importantes en el marco de la educación para la paz, como la prevención del acoso escolar, la participación en política, la memoria histórica, los dilemas morales, la participación en proyectos de impacto social y la reflexión en torno a los acuerdos de paz, entre otros. A pesar de las dificultades en el proceso de implementación, la Cátedra de la Paz ha sido bien acogida por educadores de diferentes áreas, quienes evidencian el trabajo asociado al fomento de una cultura de paz, fortaleciendo en los estudiantes el uso del diálogo para dar solución a los conflictos, el trabajo en equipo y la comunicación asertiva.

En lo referente a las competencias ciudadanas, se puede concluir que, aunque se evidencian cambios positivos en el comportamiento de los estudiantes, es prematuro asociarlos al desarrollo de esta asignatura debido a su incipiente estado en las instituciones. Pues a pesar de que la formación ciudadana es uno de los objetivos centrales de la cátedra de la paz, es también una iniciativa propuesta en los Estándares Básicos de Competencias Ciudadanas, por lo que se ha venido trabajando de manera trasversal en las diferentes áreas del currículo. Este trabajo pedagógico, aunado al desarrollo de la cátedra de la paz, ha arrojado algunos avances en el comportamiento de los estudiantes, evidentes en el trato respetuoso hacia los demás, el uso del diálogo para solucionar los conflictos, el control de las emociones, el comportamiento dentro de las instituciones y el reconocimiento y cumplimiento de las normas contempladas en los manuales de convivencia.

En relación con las estrategias de solución de conflictos utilizadas en las instituciones educativas se puede concluir que dentro del contexto escolar se fomenta la cultura del diálogo para resolver los problemas que se presentan. Los docentes se convierten en mediadores para solucionar los conflictos entre estudiantes, tratando de comprender las causas que generaron la situación, motivándolos a establecer acuerdos. No obstante, esta mediación se hace de manera espontánea, no se lleva un registro de las acciones propuestas ni se hacen seguimientos, para evitar que trasciendan a problemas mayores.

Los miembros de las comunidades educativas reconocen el Comité de Convivencia Escolar como instancia para resolver los conflictos que revisten cierto grado de gravedad, 
sin embargo, consideran que las decisiones de éste están más enfocadas a imponer sanciones que a comprender los problemas y a lograr acuerdos entre las partes implicadas. Sin embargo, en los contextos escolares o entre miembros de la comunidad educativa, son poco frecuentes las agresiones y el uso de la violencia, a pesar de estar ubicados en una zona azotada por la violencia y el conflicto armado, lo que evidencia un gran avance en esta materia.

Finalmente, en lo concerniente a la convivencia escolar se puede decir que en las instituciones educativas objeto de estudio, son escasas las actividades de promoción de la sana convivencia y prevención de la violencia escolar. Desde los comités de convivencia, no se encuentran definidas unas políticas claras sobre estos componentes. Las actividades realizadas surgen en la mayoría de los casos como iniciativas personales o acciones enmarcadas dentro de proyectos pedagógicos, que si bien favorecen la sana convivencia no generan el impacto deseado. A pesar de esto, en las instituciones prima un clima de respeto y apoyo mutuo entre directivos, docentes, estudiantes y padres de familia. Las relaciones que se establecen entre ellos son cordiales y basadas en el buen trato. Los conflictos que se presentan normalmente son entre compañeros de clases, por razones como uso de sobrenombre, indirectas o malentendidos, pero por lo general se solucionan con la mediación del director de grupo o de otro docente.

\section{REFERENCIAS BIBLIOGRÁFICAS}

Bernárdez, E. (1995). El papel del léxico en la organización textual. Madrid: Universidad Complutense de Madrid.

Bueno-Cipagauta, M. (2017). Políticas públicas de reconciliación. Un reto posible en la construcción y la sostenibilidad de la paz en Colombia. Análisis político, (90), 3-24. DOI: https://doi. org/10.15446/anpol.v30n90.68301

Cabezudo, A. (2012). Educación para la paz, una construcción de la memoria, la verdad y la justicia. Desafío pedagógico de nuestro tiempo en América Latina. Ciências Sociais Unisinos, 48(2), 139-145.

Castillo, J. (2003). La formación de ciudadanos en la escuela: un escenario posible. Revista Latinoamericana de Ciencias Sociales, Niñez y Juventud, 1(2), 1-19.

Cebreiro, B. \& Fernández, M. (2004). Estudio de casos. En F. Salvador Mata, J. L. Rodríguez Diéguez y A. Bolívar Botia, Diccionario enciclopédico de didáctica. Málaga: Aljibe.

Cerdas, E. (2013). Educación parar la paz: Fundamentos teóricos, epistemológicos y axiológicos. Revista Latinoamericana de Derechos Humanos. Volumen 24(1-2), 189-201.

Creswell, J. W. (2009). Research design: qualitative, quantitative and mixed methods approaches. Thousand Oaks, CA: Sage.

Delle Donne, A. (2018). El camino hacia una gestión de la integridad. Buenos Aires: Ucema.

Fierros, C. (2013). Convivencia inclusiva y democrática. Una perspectiva para gestionar la seguridad escolar. Sinéctica, (40), 1-18.

Fisas, V. (2006). Cultura de paz y gestión de conflictos. Barcelona: Icaria Editorial S. A.

Galtung, J. (1996). Peace by peaceful means: Peace and conflict development and civilization. Oslo: International peace Research Institute.

García, J. González, M. y Ballesteros V. (2001). Introducción a la investigación en educación (Tomo I). Madrid: UNED.

González, A. (2019). Caracterización y análisis de la implementación de la Ley 1732 de 2014 Cátedra de la Paz. Recuperado el 8 de septiembre de 2019 del sitio web: https://ciencia.lasalle. edu.co/lic_lenguas/879. 
Hernández, A. (2016). Educar para la paz y asambleas de grupo. En V. Ocejo y A. Hernández. (coords). Derechos humanos y educación para la paz (pp. 79-103). Ciudad de México: Universidad Iberoamericana.

Hoyos, V. G. (2008). Ciudadanías en formación. Bogota: Civitas Magisterio.

Jarés, X. (1999). Educación para la paz. Su teoría y la práctica. Madrid: Editorial Popular, S.A.

Johnson, R. \& Onwuegbuzie, A. (2004). Mixed methods research: a research paradigm whose time has come. Educational Research, 33(7), 14-26.

Jojoa, J. (2016). Cátedra de paz: un compromiso con la educación en derechos humanos. Nova et Vetera, (25), 87-98.

Macip, A. (2014). Formación ciudadana bajo el camino Sofía (7 áreas, 9 niveles). Recuperado el 8 de septiembre de 2019 del sitio web: https://es.slideshare.net/acacioedmundodevoll/libro-bsicoformacin-ciudadana-bajo-el-camino-de-sofia-agosto-del-14.

Martínez, V. (2001). Filosofía para hacer las paces. Barcelona: Editorial Icaria.

Ministerio de Educación Nacional. (2014). Ley 1732 de 2014. Bogotá: Ministerio de Educación Nacional.

. (2015). Decreto 1038. Bogotá: Ministerio de Educación Nacional.

Ospina, J. (2010). La educación para la paz como propuesta Ético-política de emancipación democrática. Origen, fundamentos y contenidos. Universitas. Revista de Filosofía, Derecho y Política, (11), 93-125.

Padilla, J. (2016). Derechos humanos y educación en y para los derechos humanos. En V. Ocejo y A. Hernández. (coords). Derechos humanos y educación para la paz (pp. 23-46). Ciudad de México: Universidad Iberoamericana.

Parra, K. (2014). El docente y el uso de la mediación en los procesos de enseñanza y aprendizaje. Revista de Investigación, 38(83), 155-180.

Parra, L. (2016). Transformación creativa de los conflictos. En V. Ocejo y A. Hernández. (coords). Derechos humanos y educación para la paz (pp. 47-77). Ciudad de México: Universidad Iberoamericana.

Pérez-Sauceda, J. (2015). Cultura de paz y resolución de conflictos: la importancia de la mediación en la construcción de un estado de paz. Ra Ximhai, 11(1), 109-131.

Redon, S. (2010). La escuela como espacio de ciudadanía. Estudios Pedagógicos 36(2), 213-239. http://dx.doi.org/10.4067/S0718-07052010000200013

Rodríguez, D. \& Arango, X. (2017). Despolitización y ética pública. La crisis del pensarse en colectivo en la modernidad líquida. Justicia, (31), 65-86. http://dx.doi.org/ 10.17081/just.22.31.2599

Ruiz, M., Jaraba, B. \& Romero, L. (2005). Competencias laborales y la formación universitaria. Psicología desde el Caribe, (16), 64-91.

Sacristán, J. G. (2001). Educar y convivir en la cultura global. Madrid: Editorial Morata.

Sandoval, M. (2014). Convivencia y clima escolar: claves de la gestión del conocimiento. Revista Última década, (41). 153-178. http://dx.doi.org/10.4067/S0718-22362014000200007

Servan, P. \& Servan, I. (2010). Intervención en la familia. Estudio de casos. En G. Serrano (Coord.). Modelo de investigación cualitativa en educación social y animación sociocultural: animaciones prácticas (pp. 221-252). Madrid: Narcea.

UNESCO. (2005). Hacia las sociedades del conocimiento. Informe mundial de la UNESCO. Paris: Ediciones UNESCO.

UNICEF. (2002). UNICEF va a la escuela para construir una cultura de paz y solidaridad. Buenos Aires: UNICEF oficina de Argentina. 